\title{
MULTIMODAL REPRESENTATION OF SPACE IN THE POSTERIOR PARIETAL CORTEX AND ITS USE IN PLANNING MOVEMENTS
}

\author{
Richard A. Andersen, Lawrence H. Snyder, David C. Bradley, \\ and Jing Xing \\ Division of Biology, California Institute of Technology, Pasadena, California 91125 \\ KEY WORDS: eye movements, navigation, monkey, spatial representation, optic flow
}

\begin{abstract}
Recent experiments are reviewed that indicate that sensory signals from many modalities, as well as efference copy signals from motor structures, converge in the posterior parietal cortex in order to code the spatial locations of goals for movement. These signals are combined using a specific gain mechanism that enables the different coordinate frames of the various input signals to be combined into common, distributed spatial representations. These distributed representations can be used to convert the sensory locations of stimuli into the appropriate motor coordinates required for making directed movements. Within these spatial representations of the posterior parietal cortex are neural activities related to higher cognitive functions, including attention. We review recent studies showing that the encoding of intentions to make movements is also among the cognitive functions of this area.
\end{abstract}

\section{INTRODUCTION}

The early anatomists and neurologists considered the posterior parietal cortex a classic "association" area that combined information from different sensory modalities to form a unified representation of space (for review, see Critchley 1953, Mountcastle et al 1975, Hyvärinen 1982). They did not, however, understand how this amazing feat was accomplished. Nor was it understood how, once the locations of stimuli in the environment had been specified, the motor 
cortical areas used this information to select stimuli and plan movements. There has been a recent explosion of studies that are shedding new light on how a multimodal representation of space is formed in the parietal lobe. This spatial representation is formed from a variety of modalities, including vision, somatosensation, audition, and vestibular sensation. Efference copies of motor commands, probably generated in the frontal lobes, also converge on the posterior parietal cortex and provide information about body movements. These sensory and efference copy signals are derived from a number of different neural systems and are coded in quite different coordinate frames from one another. All these signals are combined in a systematic fashion in the posterior parietal cortex to form a distributed representation of space. This distributed representation has the interesting feature that it can be used to construct multiple frames of reference, which can then be used by motor structures to code appropriate movements. A mechanism by which stimuli can be selected and plans can be made for movements also exists within this spatial representation. Old concepts about the brain being divided into sensory and motor areas need to be modified to include a third class of areas that have highly cognitive functions related to attention, intention, and decisions. Thus there is an intermediate stage between the sensory and motor structures that contains an abstract representation of space and the mental operations important for planning movements.

\section{POSTERIOR PARIETAL AREAS}

The posterior parietal cortex contains several distinct cortical areas. Those of interest here are area 7a, the lateral intraparietal area (LIP), the medial superior temporal area (MST), area 7b, and the ventral intraparietal area (VIP). Although these areas have diverse functions and use a variety of sensory modalities, they have in common the ability to process information about spatial relationships. Much of this review focuses on LIP and MST, since they have been extensively studied; however, many of the principles discussed have a general significance in the posterior parietal cortex.

One of the best understood posterior parietal areas, in functional terms, is LIP. It receives strong direct projections from extrastriate visual areas and projects to areas in the cortex and brain stem concerned with saccadic eye movements (Lynch et al 1985, Asanuma et al 1985, Blatt et al 1990). LIP cells have presaccadic responses, and electrical stimulation of the area evokes saccadic eye movements (Mountcastle et al 1975, Andersen et al 1987, Shibutani et al 1984, Kurylo \& Skavenski 1987, Thier \& Andersen 1996). Based on these findings, Andersen et al (1992) proposed that LIP is the "parietal eye field" specialized for visual-motor transformation functions related to saccades. 
Another well-studied area is MST. This area plays an important role in visual motion processing. MST has been subdivided into at least two distinct areas: MSTd (dorsal) and MSTl (lateral) (Desimone \& Ungerleider 1986; Saito et al 1986; Ungerlieder \& Desimone 1986a, 1986b; Komatsu \& Wurtz 1988). MSTd has large receptive fields that are often selective for complex patterns of motion, such as expansion, contraction, rotation, and spiraling motions (Saito et al 1986; Sakata et al 1985, 1986; Tanaka et al 1986; Graziano et al 1994). This area also receives signals related to smooth pursuit eye movements and vestibularly derived head pursuit signals (Kawano et al 1984, Sakata et al 1983, Newsome et al 1988). Because many of the patterns of motion to which MSTd neurons are selective occur with self-motion, it has been proposed that this area is important for navigation using motion cues (Tanaka et al 1986; Saito et al 1986; Sakata et al 1985; Duffy \& Wurtz 1991, 1995; Geesaman \& Andersen 1996; Bradley et al 1996). This area may also play a more general role in the perception of patterns of motion (Graziano et al 1994, Geesaman \& Andersen 1996). MST1 has smaller receptive fields and is thought to play a role in the selection of targets for smooth pursuit eye movements (Komatsu \& Wurtz 1988).

Area $7 \mathrm{a}$ is another largely visual area that has strong cortical connections with other visual areas; it also has connections with areas of the cortex associated with the highest cognitive functions, including the parahippocampal gyrus and cingulate cortex (see Lynch 1980, Goldman-Rakic 1988, Andersen et al 1990a,b for review). Although adjacent to LIP, area 7a does not appear to play a direct role in saccades (Barash et al 1991a), and unlike LIP, which has relatively smaller and contralateral visual receptive fields (Blatt et al 1990), area 7a has large, bilateral fields (Motter \& Mountcastle 1981).

Area $7 \mathrm{~b}$ and VIP are more intimately related to the somatosensory system, in terms of both anatomical connections and activation by somatosensory stimuli (Hyvärnen 1982, Andersen et al 1990a, Colby et al 1993). However, these areas also receive inputs from visual areas and respond to visual stimuli.

All of the areas listed above are strongly interconnected via corticocortical projections. As we see in this review, one of the likely consequences of this interconnectivity is that even areas like LIP and MST, which seem on the surface to be unimodal visual areas, can reveal their multimodal nature when probed with the right set of tasks.

\section{MULTIMODAL REPRESENTATION OF SPACE}

\section{Eye Position and Visual Signals}

Several years ago, we showed that single cells in area $7 \mathrm{a}$ and LIP receive a convergence of eye position and visual signals (Andersen \& Mountcastle 1983, Andersen et al 1985). This convergence produces cells with retinal receptive 
fields that are modulated in a monotonic fashion by the orbital position of the eyes. We described this modulation as an eye "gain field" because the eye position appeared to modulate the gain of the visual response. Representing the location of a visual target with respect to the head requires a convergence of both eye and retinal position information. Intuitively one would imagine that an area representing space in a head-centered reference frame would have receptive fields that are anchored in space with respect to the head, but the cells in LIP and area 7a do not appear to use this encoding scheme. Although each neuron receives both necessary input signals, its response for head-centered stimulus location is ambiguous, since its activity can be varied by changing either the eye position or the retinal location of the stimulus. However, the activity across a population of cells with different eye position and retinal position sensitivities will have a unique pattern of firing for each head-centered location. Thus the code of head-centered location in the posterior parietal cortex appears to be carried as a distributed population code. Interestingly, when neural networks are trained to transform retinal signals into head-centered coordinates by using eye position signals, the middle-layer units that make the transformation develop gain fields similar to the cells in the parietal cortex (Zipser \& Andersen 1988). This network demonstration shows that the gain-field representation can be used to code head-centered spatial locations, and also that this representation occurs rather naturally with coordinate transformation tasks.

\section{Head Position}

To code locations of stimuli with respect to the body requires one's knowing not only where the eyes are looking, but also the orientation of the head on the body. Recent experiments have shown that about half of all area $7 \mathrm{a}$ and LIP cells that have eye gain fields also have head gain fields (Brotchie et al 1995). In these experiments, monkeys were trained to orient their direction of gaze either with head movements or eye movements. An important finding was that the eye and head gain fields were the same for individual cells (see Figure 1). In other words, the modulation of the visual signal was a function of gaze direction, independent of whether the head or eyes were used to direct gaze. Such a generalization for gaze direction suggests that these cells are part of a body-centric representation, which must extract gaze direction with respect to the body as well as location of the stimulus on the retina.

There are at least three possible sources for the head position signal: an efference copy of the command to move the head, a vestibular signal generated by the head movement, and neck proprioceptive signals generated by the change in the orientation of the head on the trunk. Brotchie et al (1995) found that even when the head was moved passively to different orientations, the head gain fields were still present. This result suggests that efference copy is not the only 
source of the head position signal. To test whether vestibular signals were a factor, the animal's entire body was rotated with a turntable. In this condition the head remains at the same orientation on the trunk, but the direction of gaze has been shifted by moving the whole animal. These rotations were performed in the dark, in order to remove visual landmarks and optic flow patterns as cues to spatial orientation. Snyder et al (1993) found that many cells in the posterior parietal cortex exhibited vestibularly derived head gain fields.

To test for proprioceptive cues, the animal's body was rotated under the head, with the head fixed relative to the world. In this case there is no vestibular cue, but there are neck proprioceptive cues. Again gain fields were found with this condition (Snyder et al 1993). Thus it can be concluded that both vestibular and proprioceptive signals contribute to the generation of head gain fields.

In the course of these experiments, we found that some cells showed no gain fields in the vestibular experiment when the animals were rotated in the dark, but these cells did have gain fields if the animals were rotated in the light and then tested in the dark. This finding implies that either the remembered location of landmarks in the room or the pattern of optic flow generated by the movement was used to generate the gain fields. This result is reminiscent of "place fields" in the rat hippocampus, which also use landmarks to code the animal's location in the environment.

The findings on the source of head position signals suggest the posterior parietal cortex can use these signals to represent space in two coordinate frames, body-centered and world-centered. The neck proprioceptive signals provide information about the location of the head with respect to the trunk and thus can contribute to the representation of body-centered locations. The vestibular signals provide information about the orientation of the head in the world and thus, combined with the orientation of the eyes in the orbits and the stimulus on the retina, can code locations in world-centered coordinates. Finally, either the landmarks or the optic flow can also code the location of targets in world coordinates.

\section{Auditory Signals}

When we hear a sound in space at the same location as a visual stimulus, we perceive the source of the two stimuli as being spatially coincident. Since spatial perception occurs so naturally, we do not intuitively recognize what a formidable task it is to bring auditory and visual information into the same coordinate frame. Visual information is in the coordinates of the eye, whereas auditory information must be computed from the intraural time, intraural intensity, and spectral cues arriving at the two ears. These cues are used to construct auditory receptive fields that are in head-centered coordinates. So, how are auditory signals in head-centric coordinates in the auditory system combined with 


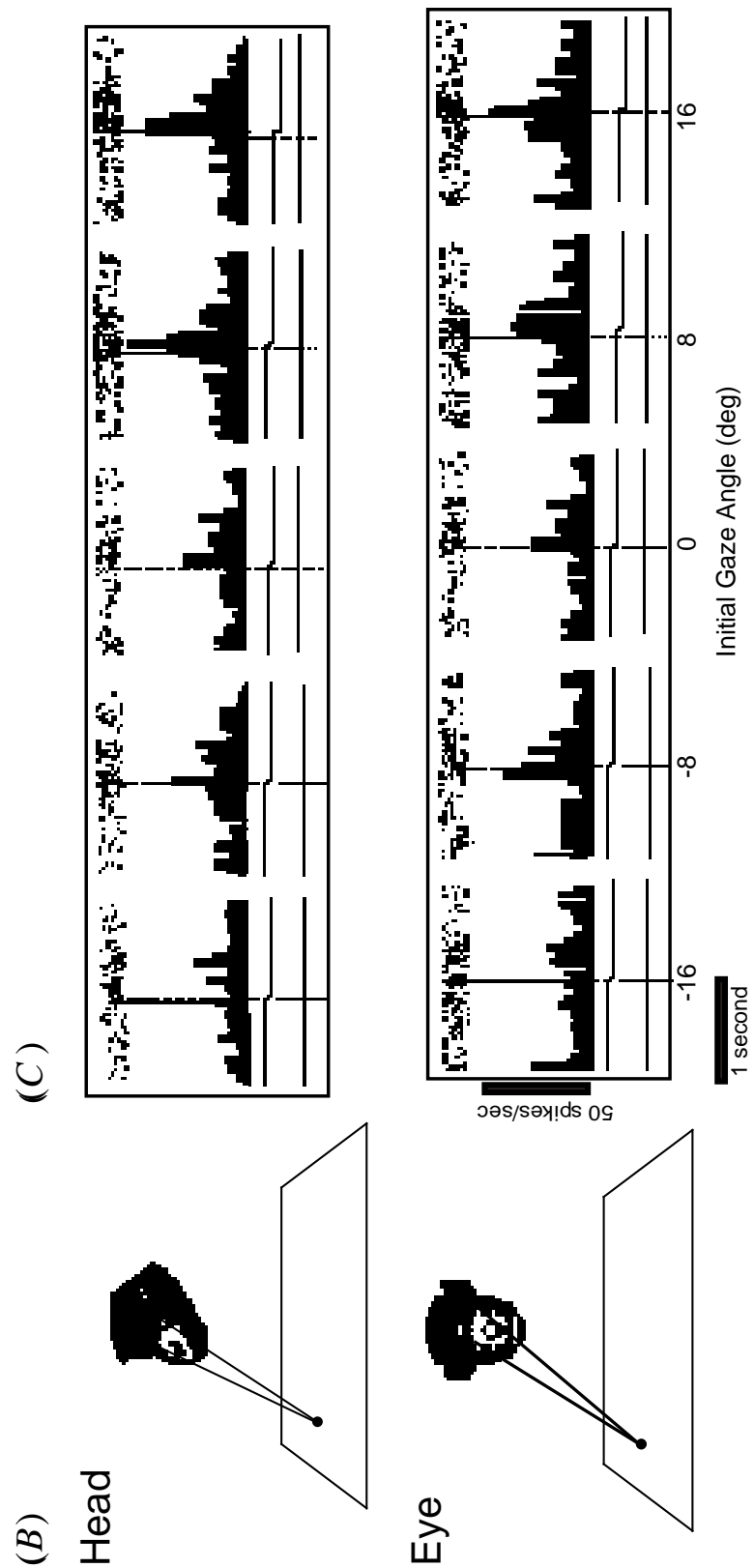

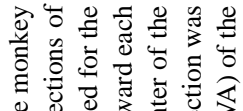

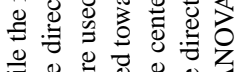
율 \% ठ

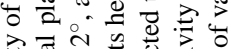

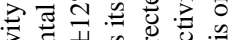

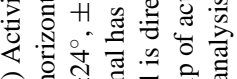

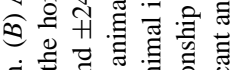

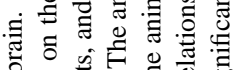
పे 0

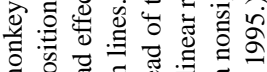

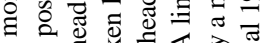
@ :

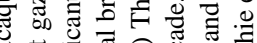

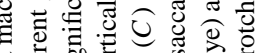
उ. to

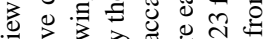

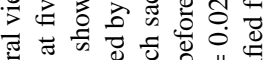

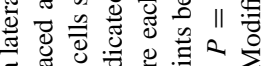

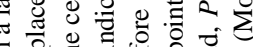

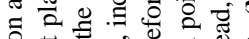

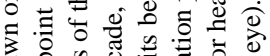

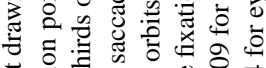

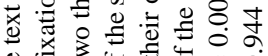

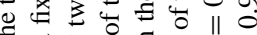

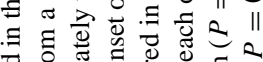
ठ

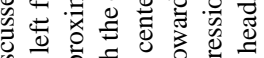

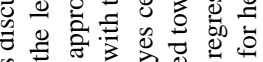

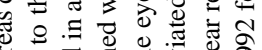

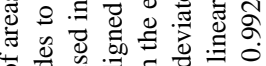

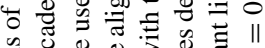

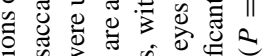
预

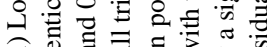

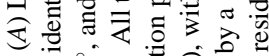

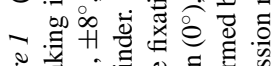

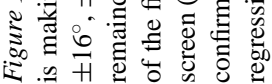
$\stackrel{\oplus}{\omega}$ 
eye-centric visual signals in the visual system? And where in the cerebral cortex does this combination occur?

As mentioned above, LIP was, until recently, believed to be a strictly visual area that processed visual targets for making saccadic eye movements. However, we can easily make eye movements to auditory as well as visual targets. Mazzoni et al (1996) recently demonstrated that when a monkey is required to memorize the location of an auditory target in the dark and then to make a saccade to it after a delay, there is activity in LIP during the presentation of the auditory target and during the delay period. This auditory response generally had the same directional preference as the visual response, suggesting that the auditory and visual receptive fields and memory fields may overlap one another (see Figure 2).

The above experiments were done when the animal was fixating straight ahead, with its head also oriented in the same direction. Under these conditions, the eye and head coordinate frames overlap. However, if the animal changes the orbital position of its eyes, then the two coordinate frames move apart. Do the auditory and visual receptive fields in LIP move apart when the eyes move, or do they share a common spatial coordinate frame? This question was addressed by testing auditory fields while the animal fixated at different orbital positions (Stricanne et al 1996). In this experiment the animals performed delayed auditory saccades in darkness, and activity was measured during the delay period between the offset of the sound and the offset of the fixation light triggering the saccade. Forty-four percent of the auditory-responding cells in LIP coded the auditory location in eye-centered coordinates - that is, auditory memory fields that actually moved with the eyes. This result is reminiscent of the superior colliculus, where auditory fields are also in eye-centered coordinates (Jay \& Sparks 1984). Another $33 \%$ of the cells were coded in head-centered coordinates, and the remaining $23 \%$ were intermediate between the two coordinate frames. Cells of all three types also had gain fields for the eye. The occurrence of cells with eye-centric auditory fields and eye gain fields suggests that at least this subpopulation shares a common, distributed representation with the visual signals in LIP.

\section{Visual Motion and Pursuit}

Although posterior parietal areas such as LIP and $7 \mathrm{a}$ are commonly discussed in terms of coordinate transformations and other spatial problems, MST has until now been seen primarily as a motion area, dealing strictly with dynamic properties of the visual field. However, the very likely involvement of this area in visual navigation (see below) implies that it also solves an important spatial problem, namely, computing the direction of self-motion in the world based on the changing retinal image. 

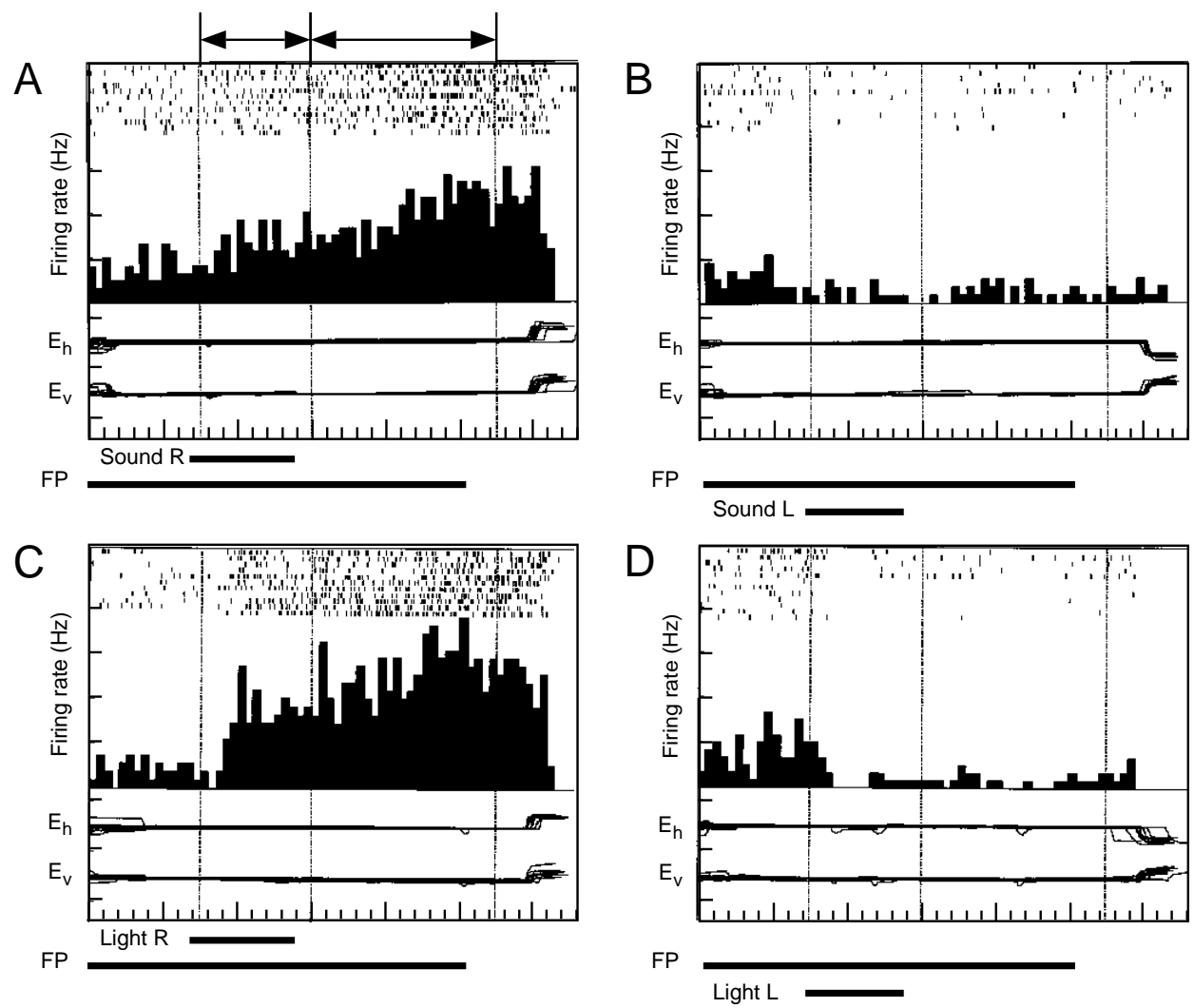

Figure 2 Activity of a neuron with auditory and visual stimulus (S)-period and memory (M)period responses. (A) Auditory memory saccade to the right. (B) Auditory memory saccade to the left. $(C)$ Visual memory saccade to the right. $(D)$ Visual memory saccade to the left. (Modified from Mazzoni et al 1996.)

Visual navigation refers to the ability to find one's heading based on visual information. A robust cue for direction of heading is the center or focus of expanding visual motion that is generated by self-motion. If a subject is moving and fixates on the horizon, the focus of expansion corresponds to the direction of heading (Gibson 1950). However, if the subject fixates and tracks a near object that is not directly ahead of him or her, then that location becomes the focus of expansion, and it no longer corresponds to the direction of heading. Interestingly, subjects can easily recover the direction of heading under these conditions (Warren \& Hannon 1988, Royden et al 1992, van den Berg 1993). 
The method by which this extraction of heading direction is achieved has been the subject of intense interest by theoreticians and psychophysicists. The flow field created by combined translation in the world by the observer and rotation of his or her eyes is a superposition of an expansion field due to observer translation and a linear motion field due to eye rotation. If the flow field can be decomposed into these two contributions, then the focus of the expansion field corresponds to the direction of heading. This is not an easy computational problem, but several researchers have suggested various algorithms and explanations of how the nervous system might perform this task using retinal cues (Lounguet-Higgins \& Prazdny 1980, Koenderink \& van Doorn 1981, Rieger \& Lawton 1985 Heeger \& Jepson 1990, Lappe \& Rauschecker 1993, Perrone \& Stone 1994).

Royden et al (1992) recently showed that the eye movement itself generates a signal, presumably an efference copy of the pursuit command, that can be used to solve this problem. If subjects make pursuit eye movements across an expanding visual stimulus, they can recover the direction of heading. However, if the identical retinal stimulus is simulated by adding linear motion to the expansion field without an eye movement, the subject cannot recover the direction of heading. These experiments indicate that information about the eye movement is a very important cue for recovering the direction of heading.

ROLE OF MSTd Cells in MSTd have properties appropriate for computing direction of heading. Early studies reported cells selective for expansion-contraction, rotation, and linear motion stimuli (Sakata et al 1985, 1986; Tanaka et al 1986; Saito et al 1986). Subsequent experiments showed that many cells were not as simple and were selective for two or even all three types of motion (Duffy \& Wurtz 1991, Graziano et al 1994).

One possible solution to the heading problem was that a decomposition was performed on the optic flow at the level of MSTd: The expansion cells were coding the motion due to observer translation, and the linear motion cells were coding the motion due to smooth pursuit. Two experiments showed this was not the case. Orban and colleagues (1992) reasoned that if this decomposition had occurred, adding a rotation to the stimulus should not affect the tuning of expansion cells. However, adding rotation detuned the expansion cells. In experiments from our lab, we developed a spiral space to test this idea (Graziano et al 1994). The spiral space had expansion-contraction along one axis and clockwise-counterclockwise rotation along the other axis. All locations between these cardinal axes represented different combinations of expansion and rotation, which are spiral motions. We reasoned that if MSTd was decomposing motion stimuli into channels of expansion, rotation, and linear motion, then all MSTd neurons should have tuning curves aligned along the cardinal axes in 
this spiral space. In fact they did not; many cells were better tuned to spiral motions than to either expansion or rotation.

In further experiments, we found that the pattern selectivity of MSTd neurons exhibited an amazing degree of position and size invariance for motion pattern selectivity (Graziano et al 1994). These cells were also form/cue invariant, responding, for instance, to a clockwise, outward spiraling stimulus regardless of whether it was a flow field, an object, or even an object made of illusory contours or non-Fourier motion (Geesaman \& Andersen 1996). These properties show that MSTd cells convey the abstract quality of a pattern of motion, e.g. rotation, much like cells in inferotemporal cortex are tuned to a static pattern, e.g. a face. This invariance for motion pattern is important for analyzing optic flow, since it indicates that information can be gathered from the entire environment or, alternatively, from a single small object and that a wide range of features can be used for recovering the motion pattern signal. Duffy \& Wurtz (1995) have shown that MSTd cells are also tuned to the retinal location of the focus of expansion. Although MSTd expansion cells are tuned for expansion all over their large receptive fields, the magnitude of response varies with the location of the focus within the receptive field. The exact focus location could therefore be computed from many neurons tuned coarsely for focus position.

MSTd SIGNALS DIRECTION OF HEADING Because MST receives a pursuit eye movement signal (Sakata et al 1983, Kawano et al 1984, Newsome et al 1988, Thier \& Erickson 1992) and has motion pattern-selective cells, it may be a brain center that computes direction of heading using optic flow and pursuit signals. Recently, we have examined how these two signals interact in MSTd. We found that MSTd cells can compensate for eye movements and code the direction of heading. In these experiments we mapped the MSTd receptive fields for their sensitivity to the location of focus of expansion. We then mapped these same receptive fields when the monkey was making smooth pursuit eye movements. We found that many MSTd neurons shift their receptive fields when the eyes are pursuing so that they more faithfully code the direction of heading than the focus of expansion on the retina. This shift does not occur when the eyes are not pursuing (Bradley et al 1996).

TEMPLATE AND COMPENSATE MODEL If we view an expanding pattern while making a pursuit eye movement to the left, the apparent focus (the focus on the retina) shifts to the left. We found that many expansion-selective MSTd neurons compensate for this by shifting their receptive field to the left (in many cases the same cell shifts its receptive field to the right to compensate for a rightward movement). This result suggests the following theory. The MSTd neurons with expansion sensitivity can be viewed as templates for optic flow 
generated by self-motion. These cells are also sensitive to the retinal location of the focus of expansion. When the eyes move, the focus tuning curve of these cells shifts in order to compensate for the retinal focus shift due to the eye movement. In this way MSTd could map out the relationship between the expansion focus and heading with relatively few neurons, each adjusting its focus preference according to the velocity of the eye. Similar models have been proposed by Perrone \& Stone (1994) and by Warren (1995), but their models require separate heading maps for different combinations of eye direction and speed (rather than a smaller number of cells that are adjusted to account for eye movement).

Two interesting features are immediately apparent from the template and compensate model. The templates should be position, size, form and cue invariant. In other words, an expansion cell should be selective for expansions everywhere in its receptive field; the strength of the response to expansion should vary from small at the edges of the receptive field to large near the center. These two features of position invariance and sensitivity to the focus location have already been documented (Graziano et al 1994, Duffy \& Wurtz 1995, Geesaman \& Andersen 1996).

A GAIN MECHANISM MAY LEAD TO COMPENSATION These experiments provide the first direct evidence that MSTd is involved in the computation of direction of heading. The method by which the pursuit compensation is achieved appears to use a gain mechanism. Approximately half the cells show the compensation effect, and it appears at first glance to be a simple shift of the receptive field. However, a more quantitative analysis of the data shows that a nonuniform gain applied to different locations in the receptive field, rather than a straight shift of the receptive field, better models the effect (Bradley et al 1996). In other words, the pursuit signal distorts the receptive field, resulting in its peak moving in the compensating direction, rather than the entire receptive field moving in the compensating direction.

There are at least two methods by which this nonuniform gain could be accomplished. In the first method the output of an MSTd neuron is computed as a logistic function of the sum of the eye velocity and retinal focus-location inputs. For cells with sigmoidally shaped focus-tuning fields, the nonlinear input-output function will cause a distortion and apparent shift in the receptive field when the eye velocity input changes. A second method requires two steps. Many MST neurons that do not compensate do have uniform gain fields; for instance, a leftward pursuit may increase the gain of the response at all locations in the receptive field of a particular neuron, and a rightward pursuit may decrease the response. Two or more uniform gain field cells with different gain fields could provide input to a cell that would compensate as a result of the gain effects 
on the input cells (Bradley et al 1996). This mechanism would work particularly well for cells with Gaussian-shaped focus-tuning fields whose peaks shift during pursuit.

HEADING DIRECTION IN MOTOR COORDINATES The cells that show compensating shifts can be considered to code the direction of heading in motor coordinates. In the above experiments, measurements were made when the eyes were in approximately the same orbital positions, but in one case the eyes were moving, and in another they were not. Because all coordinate frames are aligned, we do not know if the focus is shifted to eye-centered, head-centered, bodycentered, or world-centered coordinates. For instance, MSTd neurons may code the direction of heading with respect to the eyes. In such a scenario, the retinal focus would correspond to the direction of heading with respect to the eyes if the eyes are stationary, but would have to compensate in the correct direction to code the direction of heading with respect to the eyes if the eyes are pursuing. For example, if we are looking left of our direction of heading, the retinal image would be an expansion, with its focus to the right of the fovea. However, if we track a ground point, such that the eye pursues toward the left, the retinal focus will shift to the left. In both cases (still and moving eye), the eye would have to move right in order to look in the direction of heading. Therefore, a cell coding heading in motor coordinates should maintain the same output in both conditions. It could do this by shifting its preferred focus position to the left (thus compensating for the retinal focus shift) during leftward pursuit.

Experiments have yet to determine in which coordinate frame the direction of heading is coded. If it is in an eye-centered coordinate frame, then the situation will be very similar to the auditory case, but converting a motion pattern focus (rather than an auditory stimulus) into eye-centered coordinates. Eye and head gain fields could map this direction of heading signal to other coordinate frames for appropriate motor behaviors such as walking or driving.

PERCEPTUAL STABILITY DURING PURSUIT EYE MOVEMENTS Recent experiments from our lab suggest that area MSTd is used not only for calculating direction of heading, but also more generally for providing perceptual stability during tracking eye movements. Rotation cells have a focus of rotation that is also displaced during pursuit eye movements. However, unlike expansion cells, whose focus is displaced in the direction of eye movements, the rotation foci are displaced orthogonal to eye movement direction. For instance, a rightward pursuit will cause a clockwise rotation focus to shift down and a counterclockwise rotation focus to shift up. Remarkably, we found that the focus tuning of rotation cells in MSTd shifted orthogonal to the direction of pursuit, and in the correct direction to compensate for the focus shift (Bradley et al 1996). In 
psychophysical studies we have found that subjects compensate for the shift in the retinal image of a rotation during pursuit and report the true location of its focus.

This result suggests that MSTd may perform a more general function than just calculating direction of heading. MSTd may compensate spatially for the consequences of eye movements for all patterns of motion. Because self-motion will generate a focus of expansion in the direction of heading, this focus provides a powerful cue for navigation. If MSTd provides perceptional stability, then a correction on an expansion pattern will automatically recover the true direction of heading. However, there is also a tremendous advantage in correcting for pursuit-induced shifts on a wide variety of motion patterns. For instance, if one visually pursues horizontally past a person whose gestures include rotational components, one does not have the illusion that the person's arms are moving up or down away from his or her body. Likewise, if one wants to saccade to his or her arm, since the compensation will provide the location of the arm in motor coordinates, this eye movement will go to the right vertical location. Based on this idea of perceptual stability, compensating for pursuit eye movements while navigating is just one outcome of the more general spatial stability for motion patterns that is achieved when MSTd compensates for pursuit eye movements. Also, optic flow patterns generated during self-motion often contain significant rotation. Since MSTd expansion cells respond poorly to rotation (Orban et al 1992, Graziano et al 1994), rotation cells might be required in some cases to provide information about heading. If so, they would have to correct for pursuit eye movements by shifting their receptive fields orthogonally to the pursuit direction, as in fact they do.

\section{MODELS OF COORDINATE TRANSFORMATION}

The transformation of spatial information from retinotopic to other frames of reference has been demonstrated with neural network models. These models can help us to understand how gain fields can provide the method for transforming between coordinate frames, by representing several coordinate frames simultaneously and representing spatial frames of reference in a distributed (implicit) format. These networks can transform retinal coordinates not only into head- and body-centered coordinates but also into oculomotor (eye-centered) coordinates.

\section{Transformations Between Coordinate Frames}

Zipser \& Andersen (1988) showed that when eye and retinal position signals are converted to a map of the visual field in head-centered coordinates, the hidden units that perform this transformation develop gain fields very similar 
to those demonstrated in the posterior parietal cortex. This model showed that the activities found for posterior parietal neurons could be the basis of a distributed representation of head-centered space. Neural network models that inputted head position signals, as well as eye and retinal position signals, and that mapped locations in body-centered coordinates produced gain fields for eye and head position that were approximately the same for individual neurons. This result showed that the gain fields under these conditions are a function of gaze direction and are similar to the results of recording experiments in which gaze was changed by head or eye movements.

\section{Converting Auditory and Visual Signals to Oculomotor Coordinates}

A recent model has used as inputs auditory signals, coded in head-centered coordinates, as well as eye position and retinal position signals, and this network is trained to convert these signals to motor error coordinates at the output (Xing et al 1995). This output codes the metrics of a planned movement in motor coordinates and is similar to the activity seen in the superior colliculus, frontal eye fields, and in many cases, the posterior parietal cortex. When the network is trained in this task, the middle layers develop overlapping receptive fields for auditory and visual stimuli and eye position gain fields. It is interesting that the visual signals also develop gain fields, since both the retinally based stimuli and the motor error signals are always aligned when training the network and, in principle, do not need to use eye position information. However, the auditory and visual signals share the same circuitry and distributed representation, which results in gain fields for the visual signals.

\section{Multiple Coordinate Frames in Parietal Cortex}

In the previous example, two different coordinate frames, retinal (eye-centered) and auditory (head-centered), are brought into a single common coordinate frame at the output through an intermediate, distributed coordinate representation. We have also trained networks to input retinotopic-visual, head-centered auditory, eye position, and head position signals and to output three separate representations: eye-centered, head-centered, and body-centered (Xing et al 1995). Again, the hidden layer develops eye and head gain fields, and single cells develop bimodal auditory-visual fields. This simulation is perhaps closest to what the posterior parietal cortex is doing. By using the gain field mechanism, a variety of modalities in different coordinate frames can be integrated into a distributed representation of space (see Figure 3). A unique feature of this representation is that information is not collapsed and lost. For instance, if eye position and retinal position signals were converged to create receptive fields in head-centric coordinates, the retinal location of the stimulus could 


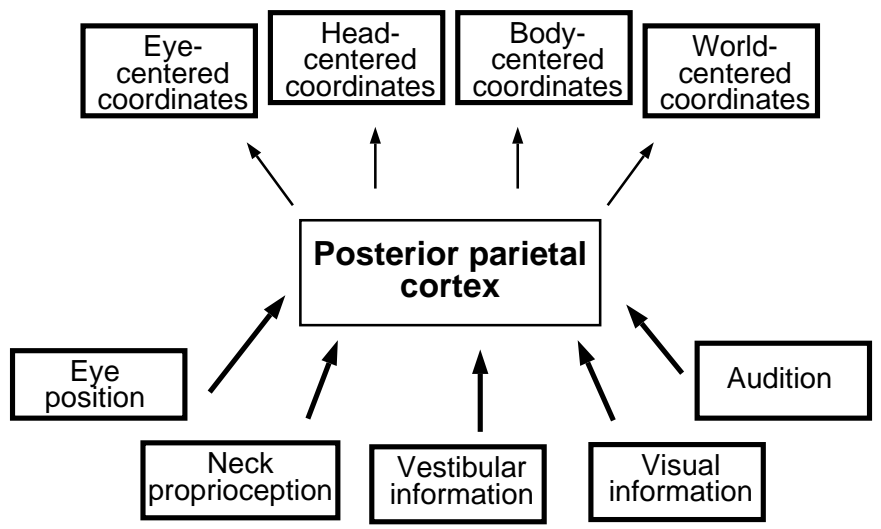

Figure 3 Posterior parietal cortex begins the transformation of retinotopic visual information into higher-order reference frames. In LIP and area $7 \mathrm{a}$, we find evidence for an intermediate stage in this process. Visual and auditory spatial information, obtained in a retinotopic and a head-centered frame of reference, respectively, is modulated by eye and head position signals. The form of this modulation is similar to that found in neural networks trained to convert from retinotopic to head-centered frames (see text for details). Head position information is obtained from both neck proprioceptive and vestibular sources. Visual allocentric information also provides a gaze in the world signal. All of these inputs-eye position, head position from neck proprioception and vestibular sources, gaze position from visual information - are used to modify retinotopic visual signals. As a result, posterior parietal cortex is positioned to provide an intermediate stage in the conversion of visual and auditory sensory information into eye-, head-, body-, and world-centered coordinate frames.

not be recovered from the distributed representation. However, the gain field mechanism retains both eye and retinal position information and can be read out by another structure, such as the superior colliculus, to create eye-centered coordinates. A simple way to envision this conversion is that cells with the same retinal receptive fields but different eye gain fields would converge on the colliculus, averaging out the eye position signal while retaining the retinal position signal. These same cells, however, could be sampled in a different way in another area, through a different pattern of connection weights, to reconstruct a receptive field in head-centered coordinates.

One implication of this distributed representation is that many coordinate frames can be represented in the same population of neurons (see Figure 3). We do not currently know if this is strictly true. For instance, about half of LIP neurons with eye gain fields also have head gain fields. This result does not necessarily indicate that there are separate populations of cells in LIP coding in head- and body-centered coordinates; when a neural network is 
trained to convert visual signals to body-centered coordinates, some units in the network have only eye gain fields. Evidence in favor of separate coordinate representations would be a strict anatomical segregation of the eye-head gain field cells and eye-only gain field cells, evidence that is so far lacking. Likewise, it is currently not known if the neck proprioceptive and vestibular gain fields are segregated; again, such an anatomical segregation would suggest separate cortical areas that coded in body- and world-centered coordinates.

The coexistence of multiple coordinate frames in the posterior parietal cortex is likely to explain why spatial deficits appear in multiple coordinate frames after lesions to this area in humans. Even if the different coordinate frames are not represented within a single distributed network, they would exist in close proximity to one another and would generally all be affected by a cortical lesion. Pouget \& Sejnowski (1995) have recently made a neural network model of parietal cortex that demonstrates just this effect-lesion to the hidden layer produces neglect in multiple coordinate frames at the output.

\section{Converting Retinotopic Signals to Oculocentric Coordinates}

When an animal plans an eye movement to a visual target, the stimulus on the retina is in the same location relative to the fovea as the goal of the eye movement planned to foveate the target. Thus no coordinate transformation is required for a simple visual saccade. However, if the eye is displaced before the eye movement, either by electrical stimulation or an intervening saccade, then the motor movement to achieve the target is different from the earlier retinal location of the stimulus. Cells in the posterior parietal cortex, frontal eye fields, and superior colliculus code the impending movement vector under these conditions, even though no visual stimulus has appeared in their receptive field (Gnadt \& Andersen 1988, Goldberg \& Bruce 1990, Mays \& Sparks 1980). Thus these cells are coding in oculomotor coordinates, which are a different coordinate frame from sensory-retinal coordinates, even though they are often aligned with one another. Krommenhoek et al (1993) and Xing et al (1995) have trained networks that convert retinal-sensory coordinates to eye-centered, oculomotor coordinates. The Krommenhoek network compensates for electrical stimulation-induced saccades prior to the eye movement. The Xing et al neural network was trained on a double-saccade task; it inputted two retinal locations and then outputted the motor vectors of two eye movements, first to one target and then to the other. In order to program the second saccade accurately, the network was required to use the remembered retinal location of the first target and update it with the new eye position. Interestingly, these networks developed eye gain fields in the hidden layer. The implication of these results is that an implicit distributed representation of head-centered location was formed in the hidden layer in order to solve the task, even though an explicit 
head-centered representation did not exist anywhere in the model. Recently $\mathrm{Li}$ et al (1995) have shown that reversible lesion of LIP produces a deficit in this double-saccade task, and this deficit appears to be largely a result of not being able to take into account eye position in the contralateral field. Such a deficit may be the result of selectively inactivating the part of the distributed representation in LIP for contralateral head (or body) centered space.

\section{Algorithms for Gain Fields}

The cellular basis of the gain field effect is of interest here. In the neural network models, the inputs to a neuron are summed, and the output is a sigmoidal (logistic) function of the inputs. This sigmoidal function enables the hidden layer to make nonlinear transformations from input to output. The gain in these networks exists in three general forms: In some cases the cells are operating close to the accelerating limb of the sigmoid, resulting in an approximate multiplication of the retinal and eye position inputs. At other times the units operate on the more linear aspect of the sigmoids, and the two inputs tend to add. Finally, some units operate around the upper saturating part of the sigmoid, and the eye and retinal position inputs show ceiling effects. All three types of gain fields are found in the recording data; combinations of the first two (both multiplicative and additive components) are quite common (Andersen et al 1990b). Other recent models of the distributed parietal representation have used only multiplications of the two inputs. Although using only a multiplication is a bit removed from the actual data, the mathematical simplification embodied in this approach has been useful in investigating the power of the distributed format for spatial representation. Pouget \& Sejnowski (1995) used basis functions formed by the multiplication of sigmoidal eye position signals and Gaussian receptive fields to show that the posterior parietal cortex could simultaneously represent locations in retinal and head-centered coordinates. Salinas \& Abbott (1995) used this same multiplication to show that accurate reaching movements can be learned by using this intermediate spatial representation and Hebbian learning.

\section{COGNITIVE INTERMEDIATES IN THE SENSORY-MOTOR TRANSFORMATION PROCESS}

Besides containing a multimodal and multiple coordinate representation of space, the posterior parietal cortex also contains circuitries that appear to be important for shifting attention, stimulus selection, and movement planning. Thus this area is an important interface between sensory cortex and motor cortex in the frontal lobes, and it performs intermediate operations of a highly cognitive nature in the sensory-motor transformation process. 


\section{Attention}

The posterior parietal cortex is intimately involved in attentional processes. Patients with lesions to this area have difficulty shifting their focus of attention (Posner et al 1984). A similar shifting role for attention is becoming apparent in recent recording experiments. Although it had been previously reported that visual signals were enhanced at the focus of attention (Bushnell et al 1981), more recent studies have reported the opposite; visual responsiveness of parietal neurons is actually reduced at the focus of attention (Steinmetz et al 1994, Robinson et al 1995). However, locations away from the focus of attention are enhanced in responsiveness, apparently signaling novel events for the shifting of attention. The reason for the discrepancy between reports is unknown, but Steinmetz \& Constantinidis (1995) point out that the earlier study used a split attention task, which might have complicated the interpretation of the results. In the middle temporal area (MT), and probably in most high-order visual parietal areas, attention can profoundly influence neuronal responsiveness and selectivity (Treue \& Maunsell 1995).

\section{Intention}

Gnadt \& Andersen (1988) described a memory-related signal in the posterior parietal cortex that was active when the monkey remembered the location of a briefly flashed stimulus and, after a delay, made a saccade to the remembered location. They further showed that if the animal performed a double-saccade task, this memory activity appeared before the second saccade that was in the motor field of the cell, even if the target had originally appeared outside the cell's receptive field. They reasoned that the cells were coding in oculomotor coordinates during the memory period of the saccade that the animal intended to make. Subsequent experiments showed that the memory-related activity was primarily a feature of LIP neurons. These LIP neurons also had bursts of activity preceding saccadic eye movements. The sensory receptive fields, memory fields, and presaccadic motor fields were generally found to overlap in eye-centered coordinates, and all three had similar eye gain fields for individual neurons. Shadlen \& Newsome (1996) have recently shown that LIP neurons become active when the animal performs a task in which it must plan a saccade in the direction it perceives a display of dots to be moving. The activity that builds up during the task prior to the eye movement is consistent with the animal planning an eye movement, although it could also reflect the direction the animal decides the stimulus is moving.

Glimcher \& Platt (PW Glimcher \& ML Platt, personal communication) have shown that when an animal is instructed to choose one of two targets for a saccade, there is more activity in LIP for the selected target. This increased activity could reflect the selection of a movement plan or the allocation of 
attention. To distinguish between these two possibilities, Glimcher \& Platt performed a variant of this task that required the animal to attend to the distractor target, which was extinguished as a cue to saccade to the selected target. This task separated the focus of attention from the selected movement. For many of the cells, the activity reflected the movement plan and not the attended location, although the activity of some cells was influenced by the attended location. The studies listed above suggest that a component of LIP activity is related to movements that the animal intends to make.

SENSORY MEMORY VS MOVEMENT PLAN Mazzoni et al (1996) recently examined the memory activity in more detail to determine if it was primarily related to intentions to make eye movements, or to a sensory memory of the location of the target. We used a delayed double-saccade paradigm in which an animal was required to memorize the location and sequence of two briefly flashed targets and then, after a delay, to make an eye movement to the first remembered location and then to the second (see Figure 4). The stimuli were configured in such a manner that in many of the trials, the second stimulus flashed in the receptive field of the cell, but the animal planned an eye movement outside the receptive field - to the first stimulus location — during the delay period. If the activity is related to attention or sensory memory, then the cell should be active during the memory period in order for the animal to attend to and remember the location of the second stimulus. However, if the memory activity codes the next saccade the animal plans to make, then the cells should have little or no activity during the delay period. Both types of cells were found, as well as cells that showed some activity for intended movement and sensory memory. However, the majority of overall activity was related to the next intended saccade and not to the remembered stimulus location (see Figure 4).

This intended movement signal was not found to be linked to the execution of an eye movement in an obligatory manner. Other experiments showed that the animals could be asked to change their plans to make an eye movement during the delay period in a memory saccade task; the intended movement activity in LIP would change in the appropriate manner with each change in plan (see Figure 5) (Bracewell et al 1996).

DO PARIETAL CELLS CODE THE TYPE OF MOVEMENT BEING PLANNED? A very definitive test of the intention signal is to show that it is contingent on the type of movement the animal plans to make. Bushnell et al (1981) performed experiments in which they recorded from posterior parietal neurons while an animal programmed an eye or reaching movement to a retinotopically identical stimulus. They claimed that the activity of the cells did not differentiate between these two types of movements. Moreover, they reported that if cells showed 

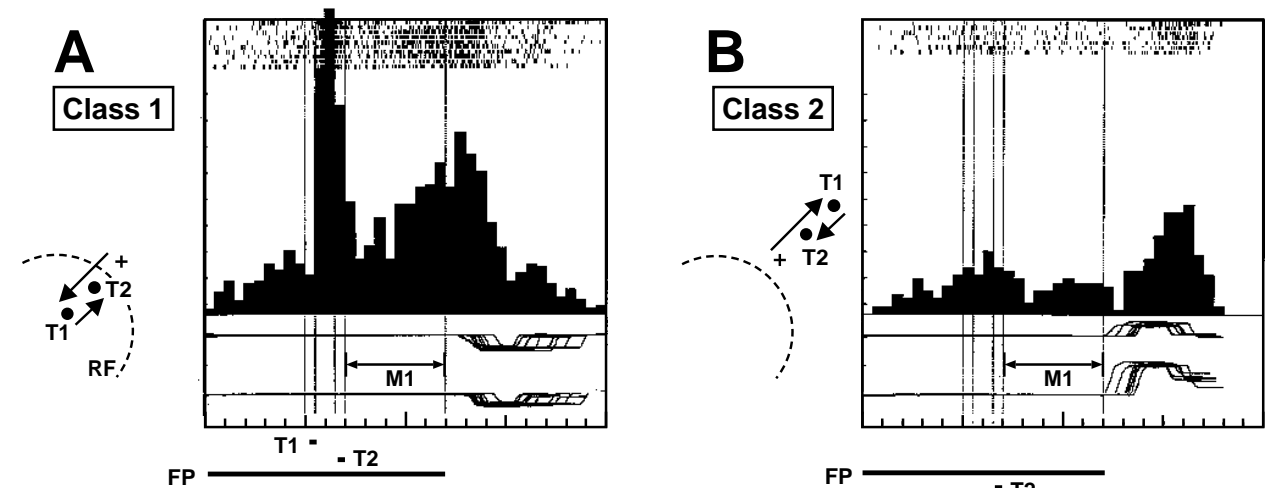

FP

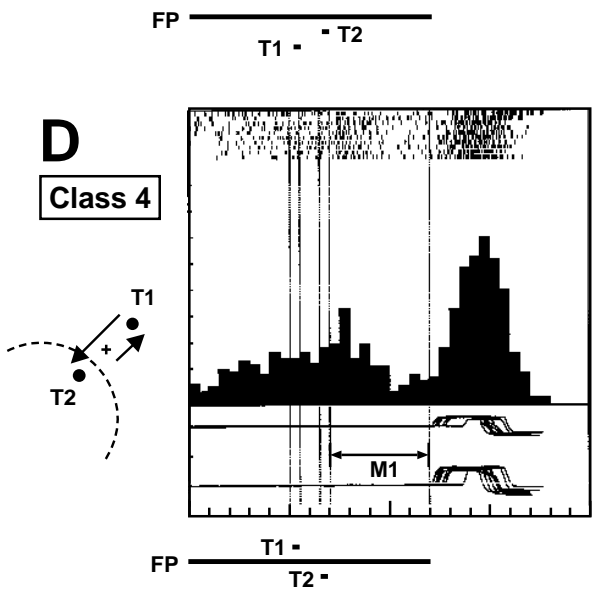

Figure 4 Activity of an LIP neuron in classes in a memory double-saccade task. Each panel has a plot that includes, (from top to bottom) the spike rasters for each trial, the time histogram (bin width $50 \mathrm{~ms}$ ) of the firing rate [ $(A-C) 20 \mathrm{~Hz} /$ division, $(D-E) 25 \mathrm{~Hz} /$ division], and the horizontal and vertical eye positions ( $30^{\circ} /$ division) (abscissa: $100 \mathrm{~ms} /$ division). The vertical dotted lines within each panel and the thick horizontal lines below each panel again show the onset and offset of the visual stimuli. The diagrams to the left of each panel show the spatial arrangement of the first and second target (T1 and T2, respectively), the first and second saccades (arrows), and the neuron's receptive field (RF). (From Mazzoni et al 1996.) 

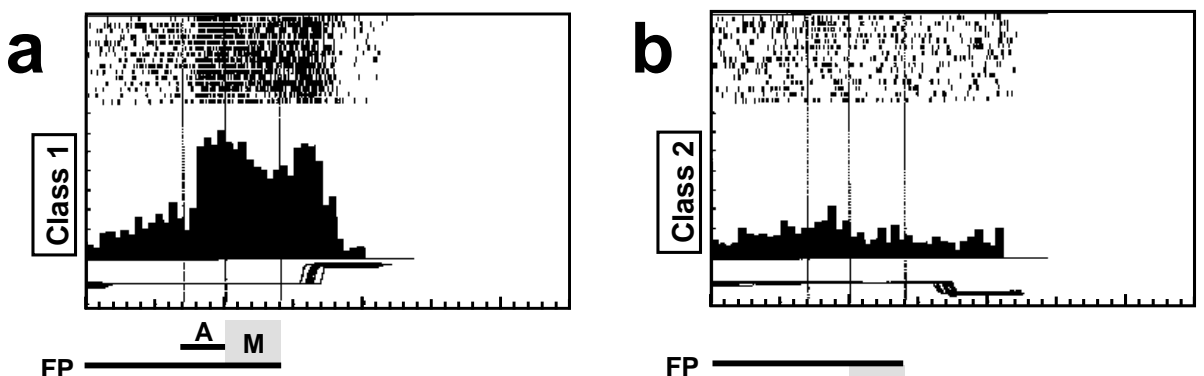

FP
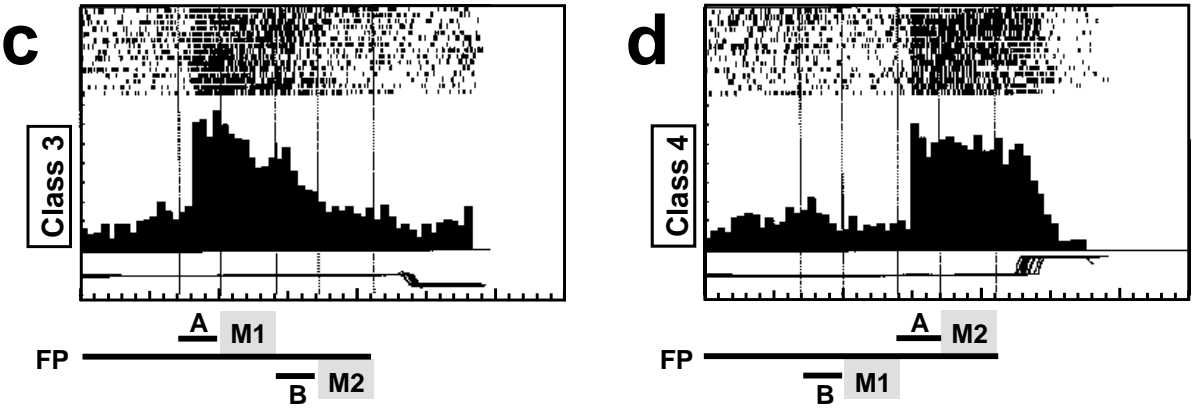

FP

A M2
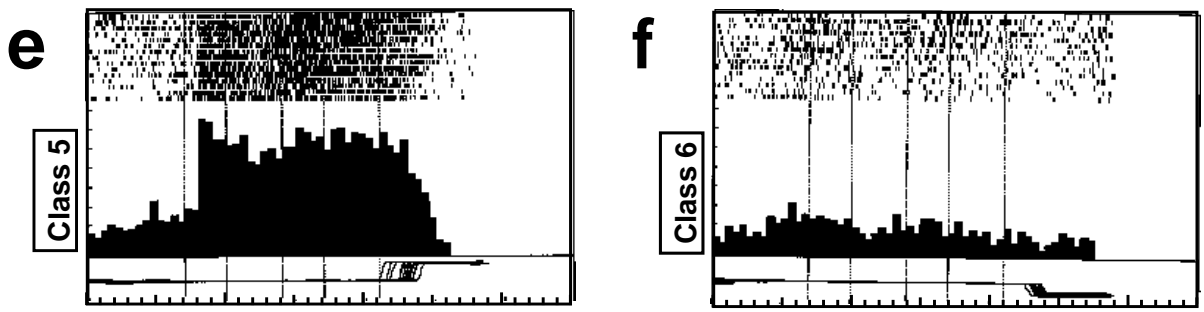

FP

A M1 A M2

FP
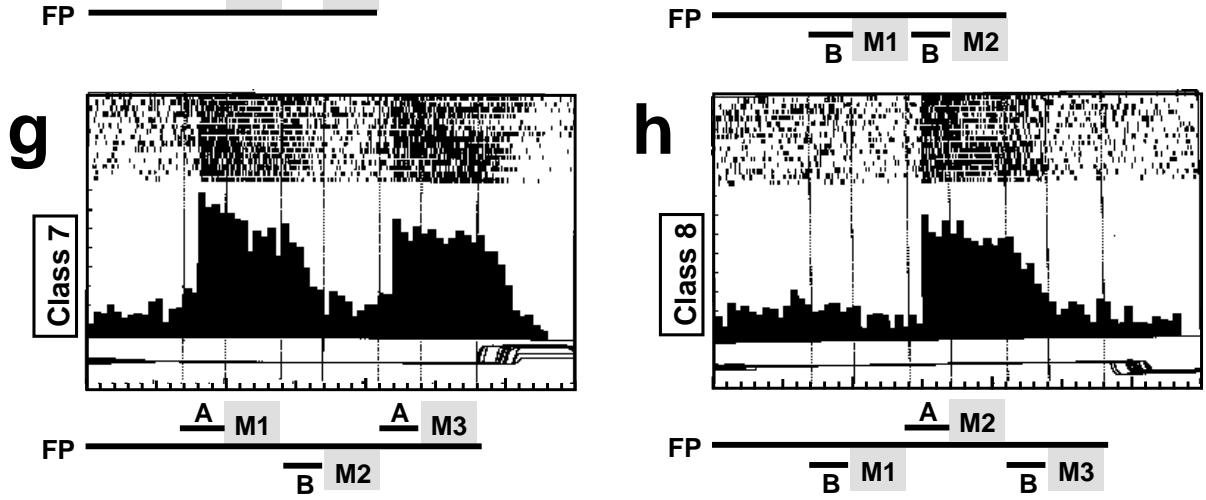
a larger response when a stimulus was a target for a movement compared with when it was not, this enhancement was the same for either a reaching or an eye movement. This result was interpreted as proof that the posterior parietal cortex is concerned with sensory location and attention and is not concerned with planning movements (Bushnell et al 1981, Colby et al 1995). We have recently repeated these experiments and found quite different results. In the task, the monkey fixates a light in a button and also presses the button with its hand. Next, a light appears briefly in the visual field, and it must remember the location of the stimulus and plan either an arm or eye movement to the stimulus, depending on its color. After a delay, the animal either makes an eye movement to the remembered location without moving the limb or vice versa. We found that two thirds of cells in the posterior parietal cortex are selective during the memory period for whether the target requires an arm or an eye movement. Interestingly, about one half of the sensory responses to the flashed targets also distinguish between the type of movement the light calls for (Snyder et al 1996). These results indicate that a good deal of activity in the posterior parietal cortex is concerned with what the animal plans to do, that is, its intentions.

\section{Intention Activity Occurs when a Monkey Considers a Movement}

An extremely important and informative result appeared as a consequence of control experiments for the above reach/eye movement task. We already knew that planning activity could occur in the absence of any overt movement. Thus when the animal was shown a target for an eye movement, it could also consider an arm movement to the target or vice versa. To control for this possibility, we had a third task in which both an eye and arm movement target appeared. The animal was required to make both movements, so both had to be programmed

Figure 5 Activity of an excitatory LIP neuron in the change-in-plan task. The abscissa in each panel represents time (100 ms/division). Each panel contains (from top to bottom) rasters of tick marks representing the occurrences of action potentials, each row corresponding to one trial; a time histogram (bin width $=50 \mathrm{~ms}$ ) of the neuron's average rate of action potential firing over all trials ( $25 \mathrm{~Hz} /$ division); and a trace of the monkey's vertical eye position ( $20^{\circ} /$ division). Onset and offset times of stimuli during the trials are indicated both by the thin vertical lines within each panel and by the thick horizontal lines below each panel. Abbreviations are as in Figure. $(a, b)$ Simple memory saccade towards the receptive field (location A, class 1) or away from it (location $\mathrm{B}$, class 2$) ;(c, d)$, single change of plan (A then B in class 3, B then A in class 4$) ;(e, f)$, no change of plan (controls) (A then A in class 5, B then B in class 6); $(g, h)$, double change of plan (A-B-A in class 7, B-A-B in class 8). Trials with one, two, and three targets were pseudorandomly interleaved so that the monkey could not predict the target sequence or the required saccade in advance. (From Bracewell et al 1996.) 
during the delay period. Usually these movements were in opposite directions. A subset of cells were found that gave activity during the memory period for both an eye and arm movement in the single-movement tasks. However, when the animal planned two movements simultaneously, and one was within the receptive field and the other was not, then the cell always coded only one of the movements. Thus, for instance, a cell might show memory-related activity for a saccade $20^{\circ}$ right or a reach movement $20^{\circ}$ right. If the animal planned an arm movement $20^{\circ}$ left and an eye movement $20^{\circ}$ right, then the cell was always inactive, whereas it was always active for the reverse set of directions (Snyder et al 1996). These dual-movement tasks needed to be performed in order to determine the true nature of a cell's activity. The results of this control experiment suggest that when a single stimulus appears, plans for both movements are represented by subpopulations of cells in the posterior parietal cortex, even though only one movement will eventually be made.

The observation that an animal can consider a movement, but subsequently not make it, may provide a unifying thread for two recent observations. Duhamel et al (1992) have shown that when two lights are flashed, and the animal makes a saccade to only one of the stimuli, activity appears after the first eye movement for the location that codes the oculomotor coordinates of the second stimulus, even if the stimulus is no longer present. This result is similar to the original double-saccade experiment of Gnadt \& Andersen (1988), with the one difference that the second saccade is not made. Based on these results, Duhamel et al (1992) have proposed that LIP codes sensory signals in retinal coordinates and that the retinal location of the remembered sensory signal has been remapped in retinal coordinates to anticipate reafference of retinal signals coding the second target after the eye movement. An alternative explanation provided by the current results is that the animal does consider making an eye movement to the second target but that this plan is not executed. We know from our hand/eye movement experiments that there are neurons in parietal cortex that code intended eye movements and not arm movements. However, these cells are active when the animal makes an arm movement and not an eye movement. Thus planning activity can occur when the animal thinks about making an eye movement, even when no eye movement is made.

Kalaska \& Crammond (1995) have shown that in a go/no go reach experiment, area 5 cells are activated by a visual stimulus during the delay period for the cell's preferred direction, regardless of whether the animal is instructed to go to the stimulus or not. Again, this result is consistent with our saccade/reach results, in which a reach cell will become active to a target in its receptive field even if an eye movement is planned there (the no go case). Thus a possible explanation of the area 5 results is that the animal thinks about making an arm movement in the cell's preferred direction, but does not execute the movement. 
In conclusion, activity in the posterior parietal cortex can be related to a plan to make a movement. When stimulus-related activity comes into the parietal cortex, it can in certain circumstances invoke more than one potential plan. Within a movement system, e.g. the eye movement system, if a potential plan is changed, then this system will change its activity to encode the most recent plan. However, some cells in another system, e.g. the reach system, may carry the plan for a limb movement, even if no limb movement is executed. In other words, these cells consider the possibility of the target for a limb movement in the absence of any other limb movement plan.

\section{SUMMARY AND CONCLUSIONS}

The posterior parietal cortex performs sensory-motor transformations. An important aspect of this transformation process is to convert between coordinate frames. In fact, at least some of the sensory-triggered activity in the posterior parietal cortex may already be coded in motor coordinates. This is certainly true of activity related to planning saccades in LIP (Gnadt \& Andersen 1988) and may also be the case for coding direction of heading in MST. This coding of signals in the coordinates of movement is consistent with the recent proposal of Goodale \& Milner (1992) that posterior parietal cortex is an action system specifying how actions can be accomplished. It is also consistent with our proposal that one of the functions of the posterior parietal cortex is to form intentions to make movements. Moreover, the posterior parietal cortex appears to represent an interface between sensory and motor areas where cognitive functions related to sensory-motor transformations such as attention, intention, and selection of targets are performed (Mountcastle et al 1975, Andersen et al 1987, Gnadt \& Andersen 1988).

As part of the sensory-motor transformation process, signals from many different modalities need to be combined in order to create an abstract representation of space that can be used to guide movements. We reviewed evidence that the posterior parietal cortex combines visual, auditory, eye position, head position, eye velocity, vestibular, and proprioceptive signals in order to perform spatial operations. These signals are combined in a systematic fashion by using the gain field mechanism. This mechanism can represent space in a distributed format that is quite powerful, allowing inputs from multiple sensory systems with discordant spatial frames and outputting signals for action in many different motor coordinate frames. Our holistic impression of space, independent of sensory modality, may be embodied in this abstract and distributed representation of space in the posterior parietal cortex. Likewise, our awareness of our internally generated wills and intentions to make movements may also be a correlate of the planning activity seen in the posterior parietal cortex. 


\section{Literature Cited}

Andersen RA, Asanuma C, Essick G, Siegel RM. 1990a. Corticocortical connections of anatomically and physiologically defined subdivisions within the inferior parietal lobule. J. Comp. Neurol. 296:65-113

Andersen RA, Bracewell RM, Barash S, Gnadt JK, Fogassi L. 1990b. Eye position effects on visual, memory, and saccade-related activity in Areas LIP and 7a of Macaque. J. Neurosci. 10(4):1176-96

Andersen RA, Brotchie PR, Mazzoni P. 1992. Evidence for the lateral intraparietal area as the parietal eye field. Curr. Opin. Neurobiol. 2:840-46

Andersen RA, Essick GK, Siegel RM. 1985. Encoding of spatial location by posterior parietal neurons. Science 230:456-58

Andersen RA, Essick GK, Siegel RM. 1987. Neurons of area 7 activated by both visual stimuli and oculomotor behavior. Exp. Brain Res. 67:316-22

Andersen RA, Mountcastle VB. 1983. The influence of the angle of gaze upon the excitability of the light-sensitive neurons of the posterior parietal cortex. J. Neurosci. 3:53248

Asanuma C, Andersen RA, Cowan WM. 1985. The thalamic relations of the caudal inferior parietal lobule and the lateral prefrontal cortex in monkeys: Divergent cortical projections form cell clusters in the medial pulvinar nucleus. J. Comp. Neurol. 235:241-54

Barash S, Bracewell RM, Fogassi L, Gnadt JW, Andersen RA. 1991a. Saccade-related activity in the lateral intraparietal area. 1. Temporal properties-comparison with area $7 \mathrm{a} . J$. Neurophysiol. 66:1095-108

Blatt GJ, Andersen RA, Stoner GR. 1990. Visual receptive-field organization and corticocortical connections of the lateral intraparietal area (area lip) in the macaque. J. Comp. Neurol. 299:421-45

Bracewell, RM, Mazzoni P, Barash S, Andersen RA. 1996. Motor intention activity in the macaque's lateral intraparietal area. II. Changes of motor plan. J. Neurophysiol. 76:1457-64

Bradley DC, Maxwell M, Andersen RA, Banks, MS, Shenoy KV. 1996. Neural mechanisms of heading perception in primate visual cortex. Science 273:1544-47

Brotchie PR, Andersen RA, Snyder LH, Goodman SJ. 1995. Head position signals used by parietal neurons to encode locations of visualstimuli. Nature 375:232-35

Bushnell MC, Goldberg ME, Robinson DL. 1981. Behavioral enhancement of visual responses in monkey cerebral cortex. I. Modulation in posterior parietal cortex related to selective visual attention. J. Neurophysiol. 46(4):755-72

Colby CL, Duhamel J-R, Goldberg M. 1993. The ventral intraparietal area (VIP) of the macaque: anatomic location and visual response properties. J. Neurophysiol. 69:90214

Colby CL, Duhamel J-R, Goldberg ME. 1995. Oculocentric spatial representation in parietal cortex. Cerebr. Cortex 5:470-81

Critchley M. 1953. The Parietal Lobes. New York: Hafner Press

Desimone R, Ungerleider LG. 1986. Multiple visual areas in the caudal superior temporal sulcus of the macaque. J. Comp. Neurol. 248:164-89

Duffy CJ, Wurtz RH. 1991. Sensitivity of MST neurons to optic flow stimuli. I. A continuum of response selectivity to large-field stimuli. J. Neurophysiol. 65(6):1329-45

Duffy CJ, Wurtz RH. 1995. Response of monkey MST neurons to optic flow stimuli with shifted centers of motion. J. Neurosci. 15(7):5192-208

Duhamel J-R, Colby CL, Goldberg ME. 1992. The updating of the representation of visual space in parietal cortex by intended eye movements. Science 255:90-92

Geesaman BJ, Andersen RA. 1996. The analysis of complex motion patterns by form/cue invariant MSTd neurons. J. Neurosci. 16:4716-32

Gibson JJ. 1950. The Perception of the Visual World. Boston: Houghton Mifflin

Gnadt JW, Andersen RA. 1988. Memory related motor planning activity in posterior parietal cortex of macaque. Exp. Brain Res. 70:21620

Goldberg ME, Bruce CJ. 1990. Primate frontal eye fields. 3. Maintenance of a spatially accurate saccade signal. J. Neurophysiol. 64:489508

Goldman-Rakic PS. 1988. Topography of cognition: parallel distributed networks in primate association cortex. Annи. Rev. Neurosci. 11:137-56

Goodale MA, Milner AD. 1992. Separate visual 
pathways for perception and action. Trends Neurosci. 15:20-25

Graziano MSA, Andersen RA, Snowden RJ. 1994. Tuning of MST neurons to spiral motions. J. Neurosci. 14(1):54-67

Heeger DJ, Jepson A. 1990. Visual perception of three-dimensional motion. Neural Comp. 2:129-37

Hyvärinen J. 1982. The Parietal Cortex of Monkey and Man. Berlin: Springer-Verlag

Jay MF, Sparks DL. 1984. Auditory receptivefields in primate superior colliculus shift with changes in eye position. Nature 309:345-47

Kalaska JF, Crammond DJ. 1995. Deciding not to go: neuronal correlates of response selection in a GO/NOGO task in primate premotor and parietal cortex. Cerebr. Cortex 5:410-28

Kawano K, Sasaki M, Yamashita M. 1984. Response properties of neurons in posterior parietal cortex of monkey during visualvestibular stimulation. I. Visual tracking neurons. J. Neurophysiol. 51:340-51

Koenderink JJ, van Doorn AJ. 1981. Exterospecific component of the motion paralax field. J. Opt. Soc. Am. 71:953-57

Komatsu H, Wurtz RH. 1988. Relation of cortical areas MT and MST to pursuit eye movements. III. Interaction with full-field visual stimulation. J. Neurophysiol. 60(2):621-44

Krommenhoek KP, Van Opstal AJ, Gielen CCAM, Van Gisbergen JAM. 1993. Remapping of neural activity in the motor colliculus: a neural network study. Vis. Res. 33(9):128798

Kurylo DD, Skavenski AA. 1987. Eye movements elicited by electrical stimulation of area PG in the monkey. J. Neurophysiol. 65:1243-53

Lappe M, Rauschecker JP. 1993. A neural network for the processing of optic flow from ego-motion in man and higher mammals. Neural Comput. 5:374-91

Li C-S, Mazzoni P, Andersen RA. 1995. Inactivation of macaque area LIP disrupts saccadic eye movements. Soc. Neurosci. 21:120.7 (Abstr.)

Lounguet-Higgins HC, Prazdny K. 1980. The interpretation of moving retinal image. Proc. R. Soc. London Ser. B 208:385-97

Lynch JC. 1980. The functional organization of posterior parietal association cortex. Behav. Brain Sci. 3:485-534

Lynch JC, Graybiel AM, Lobeck LJ. 1985. The differential projection of two cytoarchitectonic subregions of the inferior parietal lobule of macaque upon the deep layers of the superior colliculus. J. Comp. Neurol. 235:241-54

Mays LE, Sparks DL. 1980. Dissociation of visual and saccade-related responses in superior colliculus neurons. J. Neurophysiol. 43:207-32
Mazzoni P, Bracewell RM, Barash S, Andersen RA. 1996. Spatially tuned auditory responses in area LIP of macaques performing delayed memory saccades to acoustic targets. J. Neurophysiol. 75:1233-41

Motter B, Mountcastle VB. 1981. The functional properties of the light-sensitive neurons of the posterior parietal cortex studied in waking monkeys: foveal sparing and opponent vector organization. J. Neurosci. 1:3-26

Mountcastle VB, Lynch JC, Georgopoulos A, Sakata H, Acuna C. 1975. Posterior parietal cortex of the monkey: command functions for operation within extrapersonal space. $J$. Neurophysiol. 38:871-908

Newsome WT, Wurtz RH, Komatsu H. 1988. Relation of cortical areas MT and MST to pursuit eye movements. 2. Differentiation of retinal from extra retinal inputs. J. Neurophysiol. 60:604-20

Orban GA, Lagae L, Verri A, Raiguel S, Xiao D, et al. 1992. First order analysis of optical flow in monkey brain. Proc. Natl. Acad. Sci. USA 89:2595-99

Perrone JA, Stone LS. 1994. A model of selfmotion estimation within primate extrastriate visual cortex. Vis. Res. 34:2917-38

Pouget A, Sejnowski TJ. 1995. Spatial representations in the parietal cortex may use basis functions. Adv. Neural Inf. Process. 7:15764

Posner MI, Walker JA, Friedrich FA, Rafal RD. 1984. Effects of parietal injury on covert orienting of attention. J. Neurosci. 4:1863-74

Rieger J, Lawton DT. 1985. Processing differential image motion. J. Opt. Soc. Am. 2:354-59

Robinson DL, Bowman EM, Kertzman C. 1995. Covert orienting of attention in macaques. II. Contributions of parietal cortex. J. Neurophysiol. 74(2):698-721

Royden CS, Banks MKS, Crowell JA. 1992. The perception of heading during eye movements. Nature 360(6404):583-85

Saito H, Yukie M, Tanaka K, Hikosaka K. Fukada Y, Iwai E. 1986. Integration of direction signals of image motion in the superior temporal sulcus of the macaque monkey. $J$. Neurosci. 6(1):145-57

Sakata H, Shibutani H, Ito Y, Tsurugai K. 1986. Parietal cortical neurons responding to rotary movement of visual stimulus in space. Exp. Brain Res. 61:658-63

Sakata H, Shibutani H, Kawano K. 1983. Functional properties of visual tracking neurons in posterior parietal association cortex of the monkey. J. Neurophysiol. 49:1364-80

Sakata H, Shibutani H, Kawano K, Harrington TL. 1985. Neural mechanisms of space vision in the parietal association cortex of the monkey. Vision Research 25:453-63

Shadlen MN, Newsome WT. 1996. Motion per- 
ception: Seeing and deciding. Proc. Natl. Acad. Sci. USA 93:628-33

Shibutani H, Sakata H, Hyvärinen J. 1984. Saccade and blinking evoked by microstimulation of the posterior parietal association cortex of monkey. Exp. Brain Res. 55:1-8

Snyder LH, Batista A, Andersen RA. 1996. Coding the intention for an eye or arm movement in posterior parietal cortex of monkey. Soc. Neurosci. Abstr. 22:1198

Snyder LH, Brotchie P, Andersen RA. 1993. World-centered encoding of location in posterior parietal cortex of monkey. Soc. Neurosci. 19:315.11 (Abstr.)

Steinmetz MA, Conner CE, Constantinidis C, McLaughlin JR. 1994. Covert attention suppresses neuronal responses in area $7 \mathrm{a}$ of the posterior parietal cortex. J. Neurophysiol. 72:1020-23

Steinmetz MA, Constantinidis C. 1995. Neurophysiological evidence for a role of posterior parietal cortex in redirecting visual attention. Cerebr. Cortex 5:448-56

Stricanne B, Andersen RA, Mazzoni P. 1996. Eye-centered, head-centered and intermediate coding of remembered sound locations in the lateral intraparietal area. J. Neurophysiol. 76:2071-76

Tanaka K, Hikosaka K, Saito H, Yukie M, Fukada Y, Iwai E. 1986. Analysis of local and wide-field movements in the superior temporal visual areas of the macaque monkey. $J$. Neurosci. 6(1):134-44

Thier P, Andersen RA. 1996. Electrical microstimulation suggests two different forms of representation of head-centered space in the interaparietal sulcus of rhesus monkeys. Proc. Natl. Acad. Sci. USA 93:4962-67

Thier P, Erickson RG. 1992. Responses of visual tracking neurons from cortical area MSTd to visual, eye, and head motion. Eur. J. Neurosci. 4:539-53

Treue S, Maunsell JHR. 1996. Attentional modulation of direction-selective responses in the superior temporal sulcus of the macaque monkey. Nature 382:539-41

Ungerleider LG, Desimone R. 1986a. Projections to the superior temporal sulcus from the central and peripheral field representations of V1 and V2. J. Comp. Neurol. 248:147-63

Ungerleider LG, Desimone R. 1986b. Cortical connections of visual area MT in the macaque. J. Comp. Neurol. 248:190-222

van den Berg AV. 1993. The perception of heading. Nature 365:497-98

Warren WH Jr. 1995. Self-motion: visual perception and visual control. In Perception of Space and Motion, ed. W Epstein, SJ Rogers, pp. 263-325. San Diego: Academic

Warren WH, Hannon DJ. 1988. Direction of self-motion is perceived from optical flow. Nature 10(336):162-63

Xing J, Li C-S, Andersen RA. 1995. The temporal-spatial properties of LIP neurons for sequential eye movements simulated in a neural network model. Soc. Neurosci. Abstr. 21:120

Zipser D, Andersen RA. 1988. A back-propagation programmed network that stimulates response properties of a subset of posterior parietal neurons. Nature 331:67984 


\section{CONTENTS}

Patterning and Specification of the Cerebral Cortex, Pat Levitt, Mary F. Barbe, Kathie L. Eagleson

Premotor and Parietal Cortex: Corticocortical Connectivity and

Combinatorial Computations, Steven P. Wise, Driss Boussaoud, Paul B.

Johnson, Roberto Caminiti

Vertebrate Neural Induction, A. Hemmati-Brivanlou, D. Melton

THE COMPARTMENTALIZATION OF THE CEREBELLUM, Karl

Herrup and, Barbara Kuemerle

Cloned Potassium Channels from Eukaryotes and Prokaryotes, Lily Yeh

Jan, Yuh Nung Jan

The Role of Vesicular Transport Proteins in Synaptic Transmission and

Neural Degeneration, Yongjian Liu and, Robert H. Edwards

Molecular Genetic Analysis of Synaptic Plasticity, Activity- Dependent

Neural Development, Learning, and Memory in the Mamma, Chong

Chen, Susumu Tonegawa

Sleep and Arousal: Thalamocortical Mechanisms, David A. McCormick,

Thierry Bal

The Significance of Neural Ensemble Codes During Behavior and

Cognition, Sam A. Deadwyler and, Robert E. Hampson

Bcl-2 Gene Family in the Nervous System, D. E. Merry, S. J. Korsmeyer

RNA Transport, Sara Nakielny, Utz Fischer, W. Matthew Michael,

Gideon Dreyfuss

Multimodal Representation of Space in the Posterior Parietal Cortex and

Its Use in Planning Movements, Richard A. Andersen, Lawrence $H$.

Snyder, David C. Bradley, Jing Xing

Neurobiology of Speech Perception, R. Holly Fitch, Steve Miller, Paula

Tallal

Genetics of Manic Depressive Illness, Dean F. MacKinnon, Kay Redfield

Jamison, and J. Raymond DePaulo

Function-Related Plasticity in Hypothalamus, Glenn I. Hatton

375

Functional and Structural Complexity of Signal Transduction via G-

Protein-Coupled Receptors, Thomas Gudermann, Torsten Schöneberg,

Günter Schultz

ARIA: A Neuromuscular Junction Neuregulin, Gerald D. Fischbach,

Kenneth M. Rosen

429

Developmental Plasticity in Neural Circuits for a Learned Behavior,

Sarah W. Bottjer, Arthur P. Arnold

459

Pax-6 in Development and Evolution, Patrick Callaerts, Georg Halder, Walter J. Gehring

An Urge to Explain the Incomprehensible: Geoffrey Harris and the

Discovery of the Neural Control of the Pituitary Gland, G. Raisman

The Molecules of Mechanosensation, Jaime García-Añoveros, David P.

Corey

Mechanisms of Olfactory Discrimination: Converging Evidence for Common Principles Across Phyla, John G. Hildebrand, Gordon M.

Shepherd 